Revisão / Review

\title{
Lipoproteína(a) e inibição da fibrinólise na doença arterial coronariana
}

\section{Lipoprotein(a) and fibrinolysis inhibition in coronary artery disease}

Luciana M. Lima

Maria G. Carvalho

Adriano P. Sabino

Marinez O. Sousa

\begin{abstract}
O envolvimento da lipoproteína(a) nas doenças ateroscleróticas tem sido alvo de muitos estudos que têm demonstrado que esta lipoproteína é um fator de risco independente para a doença arterial coronariana (DAC). Devido à semelhança estrutural com o plasminogênio, a Lp(a) pode competir com os sítios de ligação deste, diminuindo a geração de plasmina e inibindo a fibrinólise. O trombo formado numa placa aterosclerótica rompida dispara a maioria dos eventos cardiovasculares isquêmicos. Como o trombo é dissolvido através do sistema fibrinolítico, surgiu a hipótese de que uma diminuição da atividade fibrinolítica poderia ser um fator de risco para eventos isquêmicos. Entretanto, alguns estudos demonstraram ausência de associação entre $\operatorname{Lp}($ a) e DAC, enquanto alguns atribuem o real valor preditivo à uma sub-população de $\operatorname{Lp}(a)$ com alta afinidade por fibrina. Essa hipótese sugere que alguns fenótipos de Lp(a) não estão associados à aterotrombose. Sendo assim, as pesquisas envolvendo $L p(a)$ e DAC apresentam resultados controversos, altamente dependentes da população estudada. Na população brasileira, a qual apresenta uma heterogeneidade de etnias, são raros os estudos deste tipo. Diante das notáveis controvérsias, espera-se que a presente revisão possa contribuir para suscitar ânimos no sentido da realização de estudos adicionais envolvendo a dosagem da Lp(a) em pacientes com DAC na nossa população. O conhecimento acumulado ao longo do tempo sobre a associação entre $L p(a)$ e DAC foi obtido através de estudos conduzidos fora do nosso meio. Assim, estudos fundamentados dentro da nossa realidade poderão trazer respostas mais fidedignas e adequadas para a nossa população. Rev. bras. hematol. hemoter. 2006;28(1):53-59.
\end{abstract}

Palavras-chave: Lipoproteína(a); fibrinólise; doença arterial coronariana.

\section{Aterosclerose e doença arterial coronariana}

Aterosclerose é uma doença vascular que afeta a camada íntima das artérias de médio e grande calibre. Caracteriza-se pelo acúmulo de lípides e elementos celulares, principalmente macrófagos e células musculares lisas no espaço subendotelial das artérias. ${ }^{1}$ A principal hipótese sobre a origem da aterosclerose considera que esta é uma resposta inflamatória a diferentes formas de lesão da parede vascular. $^{1,2} \mathrm{O}$ caráter crônico do processo inflamatório leva à formação de placas de ateroma, que podem progredir e obstruir as artérias.
O acúmulo de lipoproteínas plasmáticas no espaço subendotelial, especialmente lipoproteína de baixa densidade (LDL), parece ser um dos primeiros fenômenos que desencadeiam a proliferação das lesões ateroscleróticas. ${ }^{3}$ As partículas de LDL retidas no espaço subendotelial sofrem processos de oxidação e geram produtos com atividade quimiotática para os monócitos circulantes e células musculares lisas da camada média das artérias. Os monócitos são atraídos e se aderem à superfície endotelial, migram para o espaço subendotelial onde se diferenciam em macrófagos e fagocitam as partículas de LDL oxidadas presentes neste espaço. ${ }^{4}$ A captação das partículas de LDL oxidada é

Faculdade de Farmácia - Universidade Federal de Minas Gerais, Belo Horizonte, Minas Gerais, Brasil.

Correspondência para: Marinez Oliveira Sousa

Faculdade de Farmácia, Universidade Federal de Minas Gerais

Av. Antônio Carlos, 6627

31270-901 - Belo Horizonte-MG

Tel. 55.31.3499.6896 - Fax: 55.31.3499.6985

E-mail: marinez@farmacia.ufmg.br 
realizada através de receptores específicos na superfície dos monócitos, denominados receptores "scavenger", que não são submetidos à regulação. ${ }^{5}$ Desta forma, os macrófagos continuam a fagocitar as partículas de LDL oxidada e tornam-se repletos de lípides, dando origem às células espumosas, responsáveis pela produção das estrias gordurosas.

A patogênese da aterosclerose envolve vários fatores de crescimento, citocinas e outras substâncias produzidas pelas células endoteliais, células musculares lisas, macrófagos e linfócitos T, que regulam a resposta inflamatória e a proliferação celular. ${ }^{1,6} \mathrm{O}$ resultado da interação destes fatores é uma resposta fibroproliferativa que faz a estria gordurosa evoluir para uma placa aterosclerótica mais complexa. A proliferação das células musculares lisas e o acúmulo de proteínas da matriz extracelular sintetizadas e secretadas por estas células representa um importante papel na evolução da placa aterosclerótica. O tecido conjuntivo sintetizado pelas células musculares lisas forma uma capa fibrosa, que, nas lesões avançadas, cobre toda a extensão da placa. ${ }^{7}$ As síndromes coronarianas agudas estão mais relacionadas com a ruptura da placa do que com a progressão gradual da mesma. ${ }^{8}$ A ruptura ou ulceração da placa aterosclerótica provoca a formação de trombos que podem dar origem a complicações clínicas ou contribuir para o crescimento da placa de forma assintomática. A estabilidade das placas depende da espessura e composição da capa fibrosa, formada fundamentalmente por proteínas da matriz extracelular, que são sintetizadas pelas células musculares lisas (colágeno, elastina e proteoglicanos). A ruptura da capa fibrosa expõe para o fluxo sangüíneo fatores teciduais e colágeno que induzem a agregação plaquetária e a formação de trombos. ${ }^{7}$

As doenças cardiovasculares são aquelas que afetam a função do coração e dos vasos sangüíneos. ${ }^{9}$ Dentre os eventos trombóticos mais importantes estão o infarto agudo do miocárdio (IAM), o acidente vascular cerebral, a isquemia transitória e a doença arterial obstrutiva periférica (DAOP).

A doença arterial coronariana (DAC) é a causa mais comum de morte nos países desenvolvidos e em desenvolvimento em todo o mundo, ${ }^{9}$ mas tem apresentado um declínio razoável da mortalidade em países desenvolvidos, enquanto elevações relativamente rápidas e substanciais têm ocorrido em países em desenvolvimento, dentre os quais o Brasil é um dos representantes. ${ }^{10}$

Em alguns países da Europa e nos Estados Unidos, a mortalidade relacionada à aterosclerose chega a representar 50\% da mortalidade total. ${ }^{9}$ Nos grandes centros urbanos brasileiros esta é igualmente preocupante, com coeficientes de mortalidade seis a sete vezes superiores aos observados para as doenças infecciosas e parasitárias. ${ }^{11}$

A DAC constitui a causa mais importante de morbidade e mortalidade no $\mathrm{Brasil}^{8}$ e atinge, principalmente, indivíduos em idade de alta produtividade, gerando perdas econômicas significativas. Estima-se que as doenças cardiovasculares são responsáveis pela morte de 17 milhões de pessoas por ano em todo o mundo. ${ }^{9}$ A DAC é uma doença multifatorial e a prevenção desta passa pela identificação e controle, não só das dislipidemias, mas do conjunto dos fatores de risco, hereditários e adquiridos, que podem ser responsáveis diretos ou podem estar associados à doença.

A contribuição dos fatores de risco tradicionais para DAC na população em geral, como idade, sexo, diabetes mellitus, hipercolesterolemia, tabagismo e histórico familiar, surgiu dos estudos na cidade de Framingham. ${ }^{12}$ Porém, cerca de $35 \%$ dos eventos ateroscleróticos ocorrem na ausência dos denominados fatores de risco clássicos. ${ }^{13}$ Tal fato permite sugerir a possível contribuição de outros fatores de risco responsáveis por eventos vasculares até então desconhecidos. ${ }^{14-16}$

\section{Sistema fibrinolítico e doença arterial coronariana}

O sistema fibrinolítico é um sistema enzimático capaz de dissolver o coágulo sangüíneo. ${ }^{17} \mathrm{~A}$ fibrinólise consiste no mecanismo de dissolução enzimática do coágulo de fibrina que se forma no local da lesão do endotélio vascular. ${ }^{18}$ A dissolução da fibrina se faz ao mesmo tempo em que o endotélio vascular se recompõe. ${ }^{19}$

O endotélio vascular de todo o sistema cardiovascular está recoberto por uma única camada de células endoteliais justapostas que estão em contato direto com o sangue circulante. ${ }^{20}$ Estas separam o sangue da matriz subendotelial, onde se encontram proteínas adesivas importantes para a ativação da coagulação, tais como o colágeno, o fator de von Willebrand e a fibronectina. As células endoteliais têm múltiplas funções que, no seu conjunto, protegem a ativação da coagulação e a formação de trombos. ${ }^{21}$ Estas células participam da fibrinólise através da secreção de substâncias denominadas ativadores do plasminogênio, tipo tecidual (t-PA) e tipo uroquinase (u-PA), que transformam o plasminogênio em plasmina, ativando a fibrinólise ${ }^{22}$ (Figura 1). Tais células também expressam os receptores para o plasminogênio em sua superfície, sendo responsável pela geração localizada de plasmina, através da ação do t-PA ao nível da membrana celular. ${ }^{23}$

O plasminogênio, um zimogênio que normalmente está presente no plasma, é uma glicoproteína de cadeia simples sintetizada pelo fígado, ${ }^{24}$ considerada uma proenzima inativa que é convertida em plasmina, uma serinoprotease que lisa a fibrina. ${ }^{25} \mathrm{O}$ próprio endotélio controla a síntese dos ativadores do plasminogênio por meio da síntese de inibidores específicos do sistema fibrinolítico. ${ }^{26}$ Os principais inibidores da fibrinólise são o inibidor do ativador do plasminogênio tipo 1 (PAI-1), $\alpha_{2}$-antiplasmina, $\alpha_{2}$-macroglobulina e $\alpha_{1}$-antitripsina ${ }^{27}$ (Figura 2).

Há distinção na intensidade de ação dos componentes do sistema fibrinolítico dependendo do meio em que atuam, se na superfície da fibrina ou no sangue circulante. O t-PA 
apresenta alta afinidade pelo plasminogênio na superfície da fibrina e baixa afinidade no sangue circulante. ${ }^{28}$ Isso decorre da facilitação da ligação do t-PA ao plasminogênio pela fibrina. ${ }^{29} \mathrm{O}$ plasminogênio se liga ao carbono terminal da fibrina, e, desse modo, adquire uma conformação que possibilita sua maior ativação. ${ }^{30}$

A plasmina, principal enzima ativa do sistema fibrinolítico, atua em pontos específicos da molécula de fibrina, resultando em fragmentos de diferentes pesos moleculares, denominados produtos de degradação da fibrina (PDF). ${ }^{18} \mathrm{O}$ fragmento $\mathrm{X}$ é o primeiro e o maior a ser produzido, que

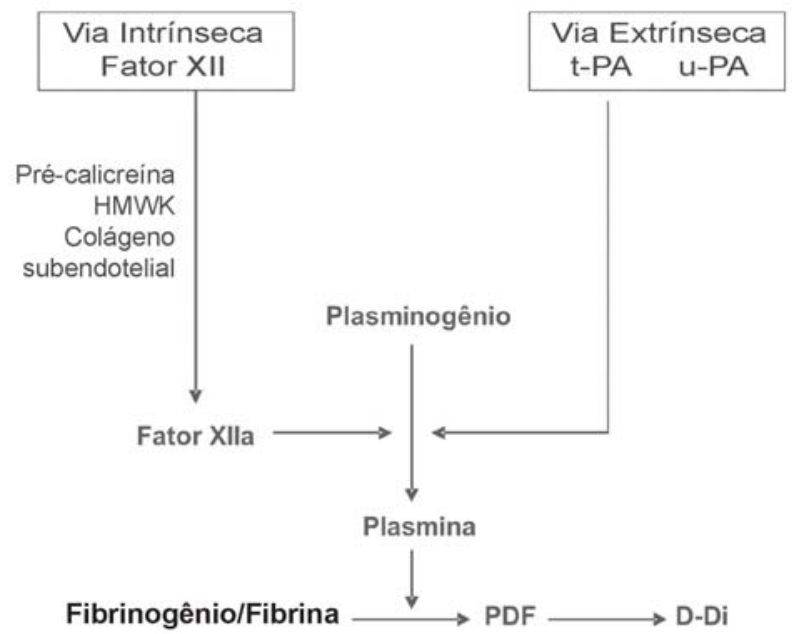

Figura 1. Representação esquemática do mecanismo de ativação do sistema fibrinolítico. As células endoteliais participam da fibrinólise através da secreção de substâncias denominadas ativadores do plasminogênio, tipo tecidual (t-PA) e tipo uroquinase (u-PA), que transformam o plasminogênio em plasmina, ativando a fibrinólise. $\mathrm{O}$ sistema fibrinolítico também pode ser ativado através da via intrínseca pela ação do fator XII ativado. HMWK = cininogênio de alto peso molecular; $\mathrm{PDF}=$ produto de degradação da fibrina; $\mathrm{D}-\mathrm{Di}=$ dímero $\mathrm{D}$

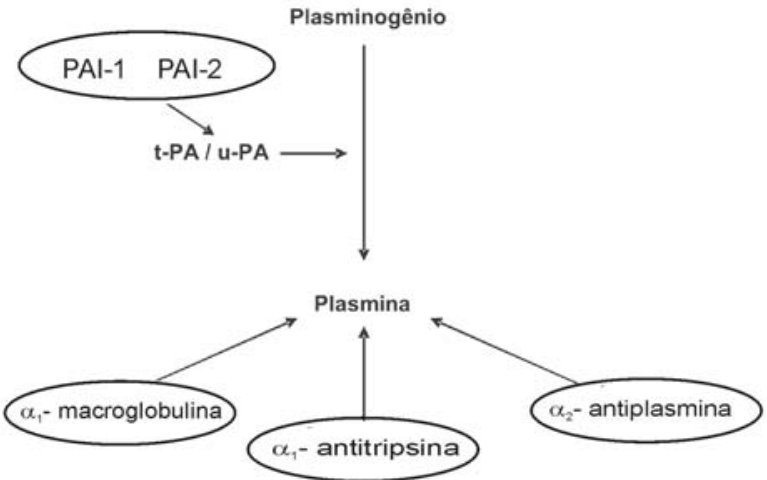

Figura 2. Representação esquemática do mecanismo de inibição do sistema fibrinolítico.O próprio endotélio controla a síntese dos ativadores do plasminogênio por meio da síntese de inibidores específicos do sistema fibrinolítico. Os principais inibidores da fibrinólise são o inibidor do ativador do plasminogênio tipo 1 (PAI-1), que atua sobre os ativadores do plasminogênio, tipo tecidual (t-PA) e tipo uroquinase (u-PA), e a $\alpha_{2}$-antiplasmina, a $\alpha_{2}$-macroglobulina e a $\alpha_{1}$-antitripsina, que atuam diretamente sobre a plasmina formada. PAl-2 = inibidor do ativador do plasminogênio tipo 2 pode ainda ser lentamente coagulado pela trombina. $\mathrm{O}$ fragmento Y e o dímero D (D-Di) têm pesos moleculares menores e não são coaguláveis. ${ }^{31}$ Os fragmentos de $\mathrm{D}(\mathrm{D}-\mathrm{Di})$ são produzidos quando a plasmina degrada a fibrina para sua remoção dos vasos sangüíneos, tecidos, ductos e líquidos orgânicos. ${ }^{30}$ Quando ocorre conversão do fibrinogênio em fibrina, aciona-se o mecanismo que mantém o balanço hemostático, com a conversão de plasminogênio em plasmina, para a rápida remoção da fibrina, prevenindo complicações trombóticas. ${ }^{32}$

A formação do trombo numa placa aterosclerótica rompida dispara a maioria dos eventos cardiovasculares isquêmicos. ${ }^{33}$ Como o trombo é dissolvido através do sistema fibrinolítico, pesquisadores levantaram a hipótese de que uma diminuição da atividade fibrinolítica poderia ser um fator de risco para eventos isquêmicos. ${ }^{34-37} \mathrm{O}$ processo fibrinolítico é localizado nos coágulos de fibrina onde o plasminogênio é depositado seletivamente nos trombos de fibrina no momento de sua formação. ${ }^{38}$ Ainda não está claro na literatura se a quantidade de plasminogênio transformado em plasmina é diretamente correlacionada com os níveis de plasminogênio. A concentração plasmática do plasminogênio não varia significativamente durante a coagulação sangüínea normal. ${ }^{38} \mathrm{O}$ PAI-1 é o inibidor primário dos ativadores fisiológicos do plasminogênio (t-PA e u-PA). O aumento do nível plasmático do PAI-1 tem sido relatado em indivíduos sobreviventes de infarto agudo do miocárdio em relação à população geral, e este aumento correlaciona-se com a recorrência do infarto. ${ }^{20}$ É de grande interesse conhecer o comportamento desse marcador em doenças que antecedem a ocorrência do infarto agudo do miocárdio, podendo contribuir para a adoção de medidas profiláticas. A contribuição da avaliação dos níveis plasmáticos de dímero D na DAC está no potencial que este marcador apresenta para avaliar o desempenho do sistema fibrinolítico, medindo a intensidade da fibrina degradada, e na sua capacidade de identificar um estado de hipercoagulabilidade. ${ }^{39}$ Níveis elevados de Dímero D são relatados não apenas em pacientes sintomáticos com aterosclerose coronariana, ${ }^{40}$ mas também em indivíduos com aterosclerose subclínica. ${ }^{41}$ Alguns autores demonstraram a associação de níveis elevados de dímero $\mathrm{D}$ com a presença da DAC, $, 2,43$ resultados que dão suporte ao conceito da contribuição da fibrina intravascular na aterotrombogênese. ${ }^{44}$

\section{Lipoproteína(a) e aterosclerose}

Desde a sua descoberta em $1963,{ }^{45}$ a lipoproteína(a) [Lp(a)] tem sido alvo de numerosas pesquisas. Esta lipoproteína apresenta uma composição lipídica similar à da LDL, com alto teor de colesterol esterificado, ${ }^{46}$ e difere no conteúdo protéico, com a presença da apolipoproteína(a) ou apo(a) ligada à apolipoproteína B através de pontes dissulfeto $^{47-50}$ (Figura 3). 


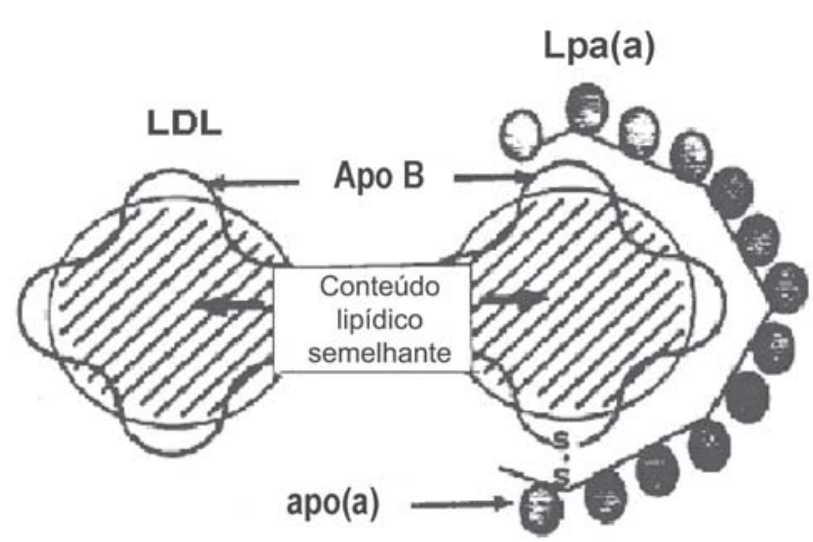

Figura 3. Semelhança estrutural entre a lipoproteína(a) $[L p(a)]$ e a lipoproteína de baixa densidade (LDL). A Lp(a) apresenta uma composição lipídica similar à da LDL, com alto teor de colesterol esterificado e difere no conteúdo protéico, com a presença da apoliproteína(a) [apo(a)] ligada à apolipoproteína B (Apo B) através de pontes dissulfeto

A apo(a) é sintetizada no fígado e a ligação com a apo B pode ocorrer tanto no espaço intracelular ou extracelular, dependendo do estado metabólico do indivíduo. ${ }^{51}$ A Lp(a) não é produto metabólico de outras lipoproteínas contendo apo B, como a lipoproteína de densidade muito baixa (VLDL), sendo, portanto, sintetizada independentemente das lipoproteínas ricas em triglicérides. ${ }^{52}$ Tal fato justificaria a observação de que a concentração plasmática da mesma não é influenciada pela dieta. ${ }^{53} \mathrm{O}$ metabolismo da $\mathrm{Lp}$ (a) não está completamente esclarecido, e os estudos demonstram que as variações de suas concentrações plasmáticas dependem mais da síntese do que do catabolismo desta lipoproteína. ${ }^{48}$ Os níveis plasmáticos de Lp(a) e a sua massa molecular são determinados geneticamente, portanto são muito variáveis entre as pessoas. ${ }^{54}$ Contudo, certas anormalidades metabólicas podem influenciar nas concentrações circulantes de Lp(a). Estes valores podem ser aumentados como parte de uma resposta de fase aguda, no diabetes mellitus, insuficiência renal crônica, síndrome nefrótica, câncer, menopausa e hipotireoidismo..$^{55}$

O aumento potencial na concentração de Lp(a) circulante como parte de uma resposta de fase aguda é de especial interesse no processo aterosclerótico, que envolve mecanismos inflamatórios, sugerindo que a concentração de Lp(a) poderia ser influenciada pela presença de doença vascular extensiva. ${ }^{55}$ Os níveis plasmáticos de Lp(a) diminuem com a falência hepática e hipertireoidismo. A concentração plasmática de Lp(a) pode ser influenciada por uma resposta de fase aguda, aumentando nas primeiras semanas após um infarto agudo do miocárdio, ${ }^{56}$ sugerindo que a associação da Lp(a) com DAC pode ser duplamente explicada: por elevadas concentrações de Lp(a) e pelo próprio processo inflamatório da aterosclerose. Sendo assim, níveis plasmáticos elevados de Lp(a) podem também ser parte da conseqüência e não somente a causa do processo aterosclerótico. ${ }^{55}$

\section{Apolipoproteína(a) e plasminogênio}

A apo(a) é uma proteína estruturalmente semelhante ao plasminogênio, devido ao variado número de repetições de seqüência de aminoácidos, homólogos à região do kringle 4 do plasminogênio. O número de kringle 4 repetido em seqüência na apo(a) de um indivíduo é geneticamente determinado, variando de 12 a 51 repetições. ${ }^{57} \mathrm{O}$ gene que codifica a apo(a) apresenta uma seqüência de 37 cópias do domínio (kringle) 4 do plasminogênio, um domínio 5 ao qual se segue o domínio da protease, ambos altamente conservados em relação ao plasminogênio ${ }^{20}$ (Figura 4). A heterogeneidade observada no peso molecular desta proteína parece estar relacionada à variabilidade do número de repetições do domínio 4. A origem da apo(a) provavelmente se deu por duplicação do gene do plasminogênio, com posteriores deleções dos éxons codificantes da região terminal e dos domínios 1 , 2 e $3 .^{58}$

A semelhança em cerca de $80 \%$ dos aminoácidos que compõem a apo(a) com o plasminogênio fornece suporte à possibilidade da Lp(a) contribuir para a trombogênese, ao competir com sítios de ligação do plasminogênio, ${ }^{59}$ diminuindo a geração da plasmina e a fibrinólise,$^{60}$ e de estabelecer uma associação entre os níveis elevados de Lp(a) com DAC. ${ }^{61}$ A Lp(a) tem sido descrita como uma variante genética ou marcador genético de alto risco para DAC. Apresenta um padrão de herança envolvendo a ação de um gene autossômico, com efeito principal na determinação de seus níveis, que sofrem, ainda, a ação de poligenes. ${ }^{62}$ Apresenta também notável heterogeneidade no peso molecular, com fenótipos variando de 280 mil a 700 mil. Diversos fenótipos de apo(a) têm sido descritos com base na sua mobilidade em gel de poliacrilamida. S1, S2, S3 e S4 são formas mais lentas, em diferentes graus. ${ }^{63} \mathrm{Em}$ um mesmo indivíduo, existe com maior freqüência apenas um fenótipo com visua-

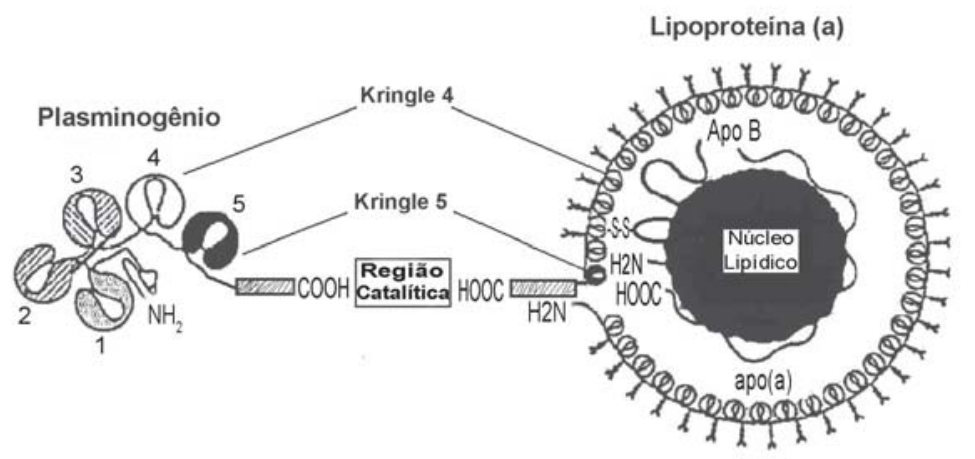

Figura 4. Semelhança estrutural entre a lipoproteína(a) e o plasminogênio. O gene que codifica a apolipoproteína(a) [apo(a)] apresenta uma seqüência de 37 cópias do domínio (kringle), 4 do plasminogênio e um domínio 5 ao qual se segue o domínio da protease, ambos altamente conservados em relação ao plasminogênio 
lização de apenas uma banda no gel de eletroforese. Os fenótipos B, S1 e S2 estão associados com altos níveis de Lp(a). Baixos níveis desta lipoproteína são observados em associação com os fenótipos S3 e S4..$^{48}$

\section{Lipoproteína(a) e doença arterial coronariana}

Em 1965, Renninger et $\mathrm{al}^{64}$ demonstraram que a concentração de Lp(a) foi significativamente mais elevada no plasma de indivíduos sobreviventes de infarto do miocárdio quando comparada com indivíduos hígidos. Esta observação foi mais tarde confirmada por outras pesquisas ${ }^{65,66}$ e a associação entre Lp(a) e DAC foi considerada estabelecida pela literatura.

Quando McLean et al (1987) ${ }^{67}$ determinaram a seqüência de aminoácidos da apo(a) e sugeriram a homologia estrutural entre esta e o plasminogênio, o verdadeiro envolvimento da $\mathrm{Lp}(\mathrm{a})$ na fisiopatologia da aterosclerose e trombose foi elucidado pelos pesquisadores. A Lp(a) é uma interação funcional entre o processo de aterosclerose e trombose, uma vez que esta participa do processo de desencadeamento da aterosclerose pela sua semelhança com a partícula de LDL, o que permite o acúmulo de Lp(a) no espaço subendotelial proporcionalmente à sua concentração plasmática..$^{48} \mathrm{~A}$ Lp(a) intacta, assim como apo(a) livre e os fragmentos proteolíticos de apo(a) foram identificados nas lesões ateroscleróticas. ${ }^{68}$ Além disso, a Lp(a) pode competir com o plasminogênio e, conseqüentemente, inibir a quebra da fibrina, gerando um estado de hipercoagulabilidade, contribuindo com a trombogênese. ${ }^{69} \mathrm{~A}$ Lp(a) está presente em altas concentrações em artérias com aterosclerose quando comparadas com artérias normais, o que contribui para a redução da atividade fibrinolítica na placa aterosclerótica. ${ }^{70}$

No entanto, recentemente, alguns estudos importantes demonstraram a ausência de associação entre níveis plasmáticos de Lp(a) e DAC, ${ }^{71}$ e o papel da Lp(a) como fator de risco para $\mathrm{DAC}$ foi novamente questionado pelos pesquisadores, enquanto outros estudos atribuíram o real valor preditivo da Lp(a) a uma subpopulação com alta afinidade por fibrina, formada por isoformas de baixo peso molecular, ${ }^{60}$ sendo as isoformas de menor tamanho e maior afinidade pela fibrina aquelas com maior associação com a DAC. ${ }^{60,72}$

No estudo de Kronenberg et al (1999) ${ }^{73}$ as isoformas de apo(a) de pequeno tamanho comportaram-se como fatores preditivos independentes de lesões ateroscleróticas avançadas (ateromatose grave) mas não de lesões recentes (ateromatose leve). Entretanto, diversos estudos, inclusive prospectivos, têm demonstrado que a Lp(a) representa um fator de risco independente para aterosclerose coronariana. ${ }^{74-77}$ Prevalece um consenso na literatura de que a $\mathrm{Lp}(\mathrm{a})$ é um fator de risco independente para DAC em diferentes populações, incluindo caucasianos, negros e orientais. ${ }^{49,78}$ Há relatos na literatura de que o valor preditivo da $\mathrm{Lp}(\mathrm{a})$ para DAC seria maior em mulheres. ${ }^{79}$
Labeur et al (1992) ${ }^{80}$ e Gupta et al (1996) ${ }^{81}$ relataram a associação entre Lp(a) e DAC confirmada por angiografia, tendo sido observado um aumento dos valores de Lp(a) com o aumento da gravidade da DAC. As pesquisas envolvendo Lp(a) e DAC apresentam resultados controversos, altamente dependentes da população estudada. Na população brasileira, que apresenta uma heterogeneidade de etnias, ${ }^{82}$ são raros os estudos deste tipo. O estudo de Lima (2005) ${ }^{83}$ confirmou a utilidade da $\mathrm{Lp}(\mathrm{a})$ como preditor da gravidade da aterosclerose coronariana, sugerindo que os níveis plasmáticos de Lp(a) devem ser determinados em pacientes com DAC, especialmente em normolipêmicos, uma vez que a Lp(a) comportou-se como um marcador de predição de gravidade da aterosclerose coronariana, independente de tabagismo, hipertensão arterial, sedentarismo, história familiar e perfil lipídico em indivíduos brasileiros. $\mathrm{O}$ autor demonstrou que as concentrações de $\mathrm{Lp}$ (a) foram significativamente aumentadas em pacientes com ateromatose grave, sendo que $64,3 \%$ destes apresentaram colesterol total e fracionado normais. $\mathrm{O}$ estudo de Maranhão et $\mathrm{al}^{84}$ demonstrou concentrações plasmáticas de Lp(a) significativamente mais elevadas em brasileiros da raça negra quando comparados com os caucasianos. No entanto, as concentrações mais altas nos indivíduos de raça negra não foram associadas com a presença ou extensão da DAC na população avaliada. Este estudo também demonstrou que níveis elevados de Lp(a) contribuíram para o desenvolvimento de DAC em caucasianos brasileiros.

Existem algumas possíveis razões para os resultados controversos encontrados pelas pesquisas envolvendo $\mathrm{Lp}$ (a) e DAC. Os fatores que afetam os níveis plasmáticos desta lipoproteína são diferentes em cada população. Hábitos nutricionais, fatores genéticos, estilo de vida e raça são fatores importantes que afetam as doenças de origem multifatorial, como é o caso da DAC. Controvérsias à parte, atualmente os consensos americano, europeu e brasileiro sobre prevenção de aterosclerose classificam a Lp(a) como fator de risco emergente e independente para DAC. . $^{13,135}$

\section{Conclusão}

Apesar da literatura indicar a existência de várias isoformas de $\mathrm{Lp}$ (a) que podem dificultar o desempenho do sistema fibrinolítico, provocando maior ou menor inibição deste, considera-se altamente relevante o envolvimento da $\mathrm{Lp}$ (a) na aterotrombose. Mesmo que as diversas isoformas de apo(a) possam afetar com intensidades diferentes o desempenho do sistema fibrinolítico, deve ser motivo de preocupação e de constante investigação a relação entre Lp(a) e DAC. Embora a triagem de indivíduos com risco de doença coronariana feita por dosagens de lípides e medidas tradicionais de fatores de risco seja vantajosa do ponto de vista econômico, as relações apontadas entre o maior risco de DAC devido à presença de marcadores genéticos de alto 
risco, como é o caso das isoformas de apo(a), pressagiam a adoção, num futuro próximo, de técnicas que possibilitem a detecção destas isoformas de maneira rotineira pelos laboratórios clínicos e possam constituir uma ferramenta potencial para auxiliar a clínica na prevenção e acompanhamento de pacientes com aterosclerose.

\begin{abstract}
The role of lipoprotein(a) $[\mathrm{Lp}(\mathrm{a})]$ in atherogenesis has been the target of many studies that have demonstrated that this lipoprotein is an independent risk factor for coronary artery disease (CAD). Due to the structural likeness with plasminogen, $L p(a)$ can compete with binding sites, reducing the plasmin generation and inhibiting fibrinolysis. Thrombus formed due to ruptured atherosclerotic plaque trigger most ischemic cardiovascular events. As the thrombus are dissolved by the fibrinolytic system, the hypothesis that a decrease in fibrinolytic activity might be a risk factor for ischemic events has been suggested. However, some studies have not demonstrated any correlation between $\operatorname{Lp}(a)$ and $C A D$ while others attribute the real predictive value to a subpopulation of $L p(a)$ with high affinity for fibrin. This hypothesis suggests that some Lp(a) phenotypes are not associated with atherothrombosis. Furthermore, research on $L p(a)$ and CAD is controversial and highly dependent on the studied population. In the Brazilian population, which presents heterogeneity of the ethnic groups, studies of this type are rare. Based on the controversies, it is expected that a contribution of the present review will motivate investigators to develop studies involving the measurement of $\operatorname{Lp}(a)$ in patients with $C A D$ in the Brazilian population. The accumulated knowledge on the association between Lp(a) and CAD was obtained through foreign studies. Thus, such studies must be performed in our setting in order to bring a better understanding for a more adequate management of CAD. Rev. bras. hematol. hemoter. 2006; 28(1):53-59.
\end{abstract}

Key words: Lipoprotein(a); fibrinolysis; coronary artery disease.

\section{Referências Bibliográficas}

1. Ross R. Mechanisms of disease: atherosclerosis an inflammatory disease. $\mathrm{N}$ Eng J Med 1999;340:115-126.

2. Libby P, Ridker PM, Maseri A. Inflammation and atherosclerosis. Circulation 2002;105:1135-1143.

3. Tousoulis D, Davies G, Stefanadis C et al. Inflammatory and thrombotic mechanisms in coronary atherosclerosis. Heart 2003;89:993-997.

4. Stary HC, Chandler AB, Dinsmore RE et al. A definition of advanced types of atherosclerotic lesions and a histological classification of atherosclerosis: a report from the Committee on Vascular Lesions of the Council on Atherosclerosis, American Heart Association. Arterioscler Thromb Vasc Biol 1994;14:840-856.

5. Beisiegel U. Lipoprotein metabolism. Eur Heart J 1998;19:S20-23. Suplemento A.

6. Pearson TA, Mensah GA, Alexander RW. Markers of inflammation and cardiovascular disease. Application to clinical and public health practice: a statement for healthcare professionals from the Centers for Disease Control and Prevention and American Heart Association. Circulation 2003;107:499-511.

7. Shin J, Edelberg JE, Hong MK. Vulnerable atherosclerotic plaque: clinical implications. Curr Vasc Pharmacol 2003;1:183-204.

8. Garcia-Palmieri MR. The endothelium in health and in cardiovascular disease. P R Health Sci J 1997;16:136-141.
9. World Health Organization. Health topics. Disponível em: <http:// www.who.int/cardiovascular_diseases/en/>.2004. Acesso em:06 nov. 2005.

10. Sociedade Brasileira de Cardiologia. III Diretrizes Brasileiras Sobre Dislipidemias e Diretrizes de Prevenção da Aterosclerose. Arq Bras Cardiol 2001;77:1-48. Suplemento III.

11. Brasil. Ministério da Saúde. Sistema de informação sobre mortalidade (SIM). Em: <http://www.datasus.gov.br>. 2001. Acesso em: 22 nov. 2005.

12. Wilson PW, D' Agostini RB, Levy B. Prediction of coronary heart disease using risk factor categories. Circulation 1998;97:1837-1847.

13. American Heart Association. NCEP Report: Implications of Recent Clinical Trials for the National Cholesterol Education Program Adult Treatment Panel III Guidelines. Circulation 2004;110:227-239.

14. Harjai KJ. Potential new cardiovascular risk factors: left ventricular hypertrophy, homocysteine, lipoprotein(a), triglycerides, oxidative stress and fibrinogen. Ann Intern Med 1999;131:376-386.

15. Ridker PM, Rifai R, Rose $L$ et al. Comparison of C-reactive protein and low-density lipoprotein cholesterol levels in the prediction of first cardiovascular events. N Engl J Med 2002;347:1557-1565.

16. Rifai N, Ridker PM. High-sensitivity C-Reactive Protein: a novel and promising marker of coronary heart disease. Clin Chem 2001;47:403-411.

17. Folsom AR, Aleksic N, Park E et al. Prospective study of fibrinolytic factors and incident coronary artery disease. TheAtherosclerosis Risk in Communities (ARIC) Study. Arterioscler Thromb Vasc Biol 2001;21:611-617.

18. Juhan-Vague I, Hans M. From fibrinogen to fibrin and its dissolution. Bull Acad Natl Med 2003;187:69-82.

19. Scazziota A, Altman R. El mecanismo de la hemostasia normal. Rev Iberoamer Thromb Hemostasia 1996;1:9-26.

20. Martinez TLR. Manual de condutas clínicas em dislipidemias. Rio de Janeiro: Medline, 2003. 392p.

21. Smith SM. Haemostatic factors and atherogenesis. Atherosclerosis 1996;124:137-143.

22. Dahlbäck B. Blood coagulation. Lancet 2000;355:1627-1632.

23. Ruschitzka FT, Noll G, Luscher TF. The endothelium in coronary artery disease. Cardiology 1997;88:3-19. Suplemento 3.

24. Jenkins GR, Seiffert D, Parmer RJ et al. Regulation of plasminogen gene expression by interleukin-6. Blood 1997;89:2.394-2.403.

25. Lijnen HR, Collen D. Mechanisms of physiological fibrinolysis. Baillieres Clin Haematol 1995;8:277-290.

26. Ploplis VA, Castellino FJ. Nonfibrinolytic functions of plasminogen. Methods 2000;21:103-110.

27. Lorenzi TF. Manual de Hematologia - Propedêutica e clínica. Rio de Janeiro: Medsi, 2003. 655p.

28. Hoffmeister A, Rothenbacher D, Khuseyinova N et al. Plasminogen levels and risk of coronary artery disease. Am J Cardiol 2002;90:1.168-1.170.

29. Tripodi A, Mannucci PM. Laboratory investigation of thrombophilia. Clin Chem 2001;47:1.597-1.606.

30. Syrovets T, Simmet T. Novel aspects and new roles for the serine protease plasmin. Cell Mol Life Sci 2004;6:873-885.

31. Matsuo T, Kobayashi H, Kario K et al. Fibrin D-dimer in thrombogenic disorders. Semin Thromb Haemost 2000;26:101-107.

32. Mohanty D, Ghosh K, Khare A et al. Thrombophilia in coronary artery disease: A double jeopardy. Indian J Med Res 2004;120:13-23.

33. Thompson SG, Kienast J, Pyke SDM et al. Hemostatic factors and the risk of myocardial infarction or sudden death in patients with angina pectoris. European Concerted Action on Thrombosis and Disabilities Angina Pectoris Study Group. N Eng J Med 1995;332:635-641.

34. Van Der Bom JG, Bots ML, Haverkate F et al. Activation products of the haemostatic system in coronary, cerebrovascular and peripheral arterial disease. Thromb Haemost 2001;85:234-239.

35. Salomaa V, Rasi V, Kulathinal S et al. Hemostatic factors as predictors of coronary events and total mortality - the FINRISK 92 hemostasis study. Arterioscler Thromb Vasc Biol 2002;22:353-358.

36. Koenig W. Haemostatic risk factors for cardiovascular diseases. Eur Heart J 1998;19:C39-43. Suplemento C. 
37. Jennings I, Cooper P. Screening for thrombophilia: a laboratory perspective. Br J Biomed Sci 2003;60:39-51.

38. Handin RI, Lux SE, Stossel TP (Ed.). Blood: principles of hematology. Philadelphia: J.B. Lippincott, 1995. 2305p.

39. Francis JL. Laboratory investigation of hypercoagulability. Semin Thromb Haemost 1998;24:111-126.

40. Danesh J, Whincuo P, Walker M et al. Fibrin D-Dimer and coronary heart disease. Prospective study and meta-analysis. Circulation 2001; 103:2.323-2.327.

41. Salomaa V, Stinson JD, Kark JD et al. Association of fibrinolytic parameters with early atherosclerosis: The ARIC Study. Circulation 1995;92:284-290.

42. Koenig W, Rothenbacher D, Hoffmeister A et al. Plasma fibrin D-Dimer levels and risk of stable coronary artery disease: results of a large casecontrol study. Arterioscler Thromb Vasc Biol 2001;21:1701-1705.

43. Lowe GDO, Rumley A, Sweetnam PM et al. Fibrin D-dimer, markers of activation and the risk of major ischaemic heart disease in the Caerphilly Study. Thromb Haemost 2001;85:822-827.

44. Tataru MC, Heinrich J, Junker R et al. D-dimers in relation to the severity of arteriosclerosis in patients with stable angina pectoris after myocardial infarction. Eur Heart J 1999;20:1.493-1.502.

45. Berg K. A new serum type system in man: the Lp system. Acta Pathol Scand 1963;59:382-386.

46. Corvilain B. Lipoprotein metabolism. Rev Med Brux 1997;19:3-9.

47. Hajjar KA, Nachman RL. The role of lipoprotein(a) in atherogenesis and thrombosis. Ann Rev Med 1996;47:423-442.

48. Koschinsky ML. Lipoprotein(a) and the link between atherosclerosis and thrombosis. Can J Cardiol 2004;20:37B-43B. Suplemento B.

49. Pati U, Pati N. Lipoprotein(a), atherosclerosis, and apolipoprotein(a) gene polymorphism. Mol Gen Met 2000;71:87-92.

50. Utermann G. The mysteries of lipoprotein(a). Science 1989;8:904-910.

51. Scanu AM. Structural and functional polymorphism of lipoprotein(a): biological and clinical implications. Clin Chem 1995;41:170-172.

52. Krempler F, Kostner GM, Bolzano K et al. Lipoprotein(a) is not a metabolic product of other lipoproteins containing apolipoprotein B. B Bioch Bioph Acta 1979;575:63-70.

3. Brown SA, Morrisett J, Patsch JR et al. Influence of short term dietary cholesterol and fat on human plasma Lp(a) and LDL levels. J Lipid Res 1991;32:1.281-1.289.

54. Marcovina SM, Koschinsky ML, Alberts JJ et al. Report of The National Heart, Lung, and Blood Institute Workshop on Lipoprotein(a) and Cardiovascular Disease: recent advances and future directions. Clin Chem 2003;49:1.785-1.796.

55. Milionis HJ, Winder AF, Mikhailidis DP. Lipoprotein(a) and stroke. J Clin Pathol 2000;53:487-496.

56. Maeda S, Abe A, Seishima M et al. Transient changes of serum lipoprotein(a) as na acute phase protein. Atherosclerosis 1989;78:145-451.

57. Graw A, Hobbs HH. Molecular genetics of lipoprotein(a): new pieces to the puzzle. Curr Opin Lipidol 1994;5:149-155.

58. Frank SL. The apolipoprotein(a) gene resides on human chromosome 6q2627 , in close proximity to the homologous gene for plasminogen. Hum Genet 1988;79:352-356.

59. Hancock MA, Boffa MB, Marcovina SM et al. Inhibition of plasminogen activation by lipoprotein(a): critical domains in apolipoprotein(a) and mechanism of inhibition on fibrin and degraded fibrin surfaces. J Biol Chem 2003;278:23260-23269.

60. Anglés-Cano E, Peña-Díaz A, Loyau S. Inhibition of fibrinolysis by lipoprotein(a). Ann NY Acad Sci 2001;936:261-275

61. Imhof A, Rothenbacher D, Khuseyinova N et al. Plasma lipoprotein Lp(a), markers of haemostasis and inflammation, and risk and severity of coronary heart disease. Eur J Cardiovasc Prev Rehabil 2003;10:362-370.

62. Rees A. The apo(a) gene: structure/function relationships and the possible link with thrombotic atheromatous disease. Br Med Bull 1990;46:873-890.

63. Koschinsky ML, Marcovina SM. Structure-function relationships in apolipoprotein(a): insights into lipoprotein(a) assembly and pathogenicity. Curr Opin Lipidol 2004;15:167-174.

64. Renninger W, Wendt GG, Nawrocki P et al. Beitrag zur problematic des Lp-systems. Humangenetik 1965;1:658-667.

65. Genest JJr, Jenner JL, Mcnamara JR et al. Prevalence of lipoprotein(a) [Lp(a)] excess in coronary artery disease. Am J Cardiol 1991;67:1.039-1.045.

66. Hopkins PN, Wu LL, Hunt SC. Lipoprotein(a) interactions with lipid and nonlipid risk factors in early familial coronary artery disease. Arterioscler Thromb Vasc Biol 1997;17:2.783-2.792.

67. McLean JW, Tomlinson JE, Kuang WJ. cDNA sequence of human apolipoprotein(a) is homologous to plasminogen. Nature 1987;330:132-137.

68. Hoff HF, O'Neil J, Yashiro A. Partial characterization of lipoproteins containing apo(a) in human atherosclerotic lesions. J lipid Res 1993; 34:789-798.

69. Schachter M. Lipoprotein(a) and cardiovascular risk. Int J Cardiol 2000;74:169-170.

70. Duriez P, Dallongeville J, Fruchart JC. Lipoprotein(a) as a marker for coronary heart disease. Br J Clin Pract 1996;77:S54-61. Suplemento A

71. Cantin B, Gagnon F, Moorjani S et al. Is lipoprotein(a) an independent risk factor for ischemic heart disease in men? The Quebec Cardiovascular Study. JACC 1998;31:519-525.

72. Peña-Díaz A, Izaguirre-Avila R, Anglés-Cano E. Lipoprotein Lp(a) and atherothrombotic disease. Arch Med Res 2000;31:353-359.

73. Kronenberg F, Kronenberg MF, Kiechal S. Role of lipoprotein(a) and apolipoprotein(a) phenotype in atherogenesis. Prospective results from the Bruneck Study. Circulation 1999;100:1.154-1.160.

74. Luc G, Bard JM, Arveiler D et al. Lipoprotein(a) as a predictor of coronary artery disease: the PRIME Study. Atherosclerosis 2002;163:377-384.

75. Danesh J, Collins R, Peto R. Lipoprotein(a) and coronary heart disease. Meta-analysis of prospective studies. Circulation 2000;102:1.082-1.085.

76. Fujino A, Watanabe T, Kunii $\mathrm{H}$ et al. Lipoprotein(a) is a potential coronary risk factor. Jpn Circ J 2000;64:51-56.

77. Rim L, Ali B, Slim BA et al. Lipoprotein(a): a new risk factor for coronary artery disease. Tunis Med 2000;78:648-652.

78. Marcovina SM, Alberts JJ, Wijsman E. Differences in Lp(a) concentrations and apo(a) polymorphs between black and white Americans. J Lipid Res 1996;37:2.569-2.585.

79. Frohlich J, Dobiásová M, Adler L et al. Gender differences in plasma levels of Lp(a) in patients with angiographically proven coronary artery disease. Physiol Res 2004;53:481-486.

80. Labeur C, De Bacquer D, De Backer G et al. Plasma lipoprotein(a) values and severity of coronary artery disease in a large population of patients undergoing coronary angiography. Clin Chem 1992;38:2.231-2.266.

81. Gupta R, Vasisht S, Bahl VK et al. Correlation of lipoprotein(a) to angiographically defined coronary artery disease in Indians. Int J Cardiol 1996;57:265-270.

82. Parra FC, Amado RC, Lambertucci Jr et al. Color and genomic ancestry in Brazilians. PNAS 2003;100:177-182.

83. Lima LM. Perfil lipídico e parâmetros hemostáticos em indivíduos submetidos à angiografia coronariana. 2005. 103f. Dissertação (Mestrado em Ciências Farmacêuticas). Faculdade de Farmácia, UFMG, Belo Horizonte.

84. Maranhão RC, Vinagre CG, Arie S et al. Lipoprotein(a) in subjects with or without coronary artery disease: relation to clinical history and risk factors. Braz J Med Biol Res 1995;28:439-46.

85. European Society of Cardiology. European guidelines on cardiovascular disease prevention in clinical practice: Third Joint Task Force of European and Other Societies on Cardiovascular Disease Prevention in Clinical Practice. Eur J Card Prev Reh 2003;10:S1-78. Suplemento 1.

Avaliação: Editor e dois revisores externos

Conflito de interesse: não declarado

Recebido: 02/02/2006

Aceito após modificações: 07/03/2006 